\title{
Lepton flavor violation from SUSY with nonuniversal scalars
}

\author{
Howard Baer, ${ }^{1, *}$ Vernon Barger, ${ }^{2, \dagger}$ and Hasan Serce ${ }^{2,+}$ \\ ${ }^{1}$ Department of Physics and Astronomy, University of Oklahoma, Norman, Oklahoma 73019, USA \\ ${ }^{2}$ Department of Physics, University of Wisconsin, Madison, Wisconsin 53706, USA
}

(Received 22 July 2019; published 14 October 2019)

\begin{abstract}
Right-handed neutrinos in supersymmetric models can act as the source of lepton flavor violation (LFV). We present experimental implications of lepton flavor-violating processes within a supersymmetric type-I seesaw framework in the three-extra-parameter nonuniversal Higgs model (NUHM3) for large [PontecorvoMaki-Nakawaga-Sakata- (PMNS-) like] and small [Cabibbo-Kobayashi-Maskawa- (CKM-) like] Yukawa mixing scenarios. We highlight LFV predictions for the natural (low $\Delta_{\mathrm{EW}}$ ) portion of parameter space. Our numerical analysis includes full two-loop renormalization group running effects for the three neutrino masses and mass matrices. We show the projected discovery reach of various LFV experiments (i.e., Mu2e, Mu3e, MEG-II, Belle-II), and specify regions that have already been excluded by the LHC searches. Our results depend strongly on whether one has a normal sneutrino ordering (NSO) or an inverted sneutrino ordering (ISO). Natural supersymmetry (SUSY) with a NSO is already excluded by MEG-2013 results while large portions of ISO have been or will soon be tested. However, LFV processes from natural SUSY with small Yukawa mixing and an ISO seem below any projected sensitivities. A substantial amount of the remaining parameter space of models with large PMNS-like neutrino mixing will be probed by Mu2e and MEG-II experiments whereas small, CKM-like Yukawa mixing predicts LFV decays which can hide from LFV experiments.
\end{abstract}

DOI: 10.1103/PhysRevResearch.1.033022

\section{INTRODUCTION}

Despite the lack of signals from the large hadron collider (LHC) [1], supersymmetry (SUSY) remains a very compelling model for physics beyond the standard model. SUSY naturally solves the big hierarchy problem [2], introduces a dark matter candidate [3], and explains neutrino masses when augmented with right-handed (RH) Majorana neutrinos [4]. So far, the minimal supersymmetric standard model (MSSM) [5] has been challenged and yet has survived four experimental tests: (1) gauge coupling unification [6], (2) discovery of the top quark with $m_{t}$ within 100-200 GeV for a successful radiative electroweak symmetry breaking [7], (3) the Higgs boson was discovered with $m_{h} \simeq 125 \mathrm{GeV}$, within the narrow range of MSSM allowed values [8], and (4) precision electroweak observables, $m_{W}$ vs $m_{t}$ plane, favor heavy SUSY even more than the standard model (SM) [9].

Regarding the neutrino sector, observations of neutrino oscillations confirm that neutrinos are massive particles [10]. Although individual masses of neutrinos are unknown, experiments can probe the mass gaps between the generations. Measured values of the mass gaps suggest two scenarios

\footnotetext{
*baer@nhn.ou.edu

†barger@pheno.wisc.edu

‡serce@ou.edu
}

Published by the American Physical Society under the terms of the Creative Commons Attribution 4.0 International license. Further distribution of this work must maintain attribution to the author(s) and the published article's title, journal citation, and DOI. for the neutrino mass hierarchy: normal $\left(m_{\nu_{3}}>m_{v_{2}} \gtrsim m_{v_{1}}\right)$ and inverted $\left(m_{v_{3}}<m_{\nu_{1}} \lesssim m_{v_{2}}\right)$. A global analysis based on oscillation and nonoscillation data within a three-neutrino framework performed by Capozzi et al. [11] with $3 \sigma$ deviations gives [12]

$$
\Delta m_{21}^{2}=\left(7.37_{-0.44}^{+0.59}\right) \times 10^{-5} \mathrm{eV}^{2}, \quad \sin \theta_{12}=0.297_{-0.47}^{+0.57}
$$

and

normal hierarchy $(\mathrm{NH})$ : inverted hierarchy (IH) :

$$
\begin{aligned}
\Delta m_{31}^{2} & =\left(2.56_{-0.11}^{+0.13}\right) \times 10^{-3} \mathrm{eV}^{2}, \\
\Delta m_{23}^{2} & =\left(2.54_{-0.11}^{+0.11}\right) \times 10^{-3} \mathrm{eV}^{2}, \\
\sin \theta_{13} & =0.0215 \pm 0.25, \quad \sin \theta_{13}=0.0216 \pm 0.26, \\
\sin \theta_{23} & =0.425_{-0.044}^{+0.19}, \quad \sin \theta_{23}=0.589_{-0.205}^{+0.047},
\end{aligned}
$$

where $\Delta m_{i j}^{2} \equiv m_{i}^{2}-m_{j}^{2}$. Analyses based on recent observations from IceCube and NOvA favor the normal neutrino mass hierarchy [13,14].

In a more recent study [15], it has been shown that current neutrino data can only have a good $\chi^{2}$ fit for SO(10)-based models when SUSY threshold corrections are included. The analysis is carried out using Pontecorvo-MakiNakawaga-Sakata (PMNS) mixing and the best-fit points give $m_{\nu_{1}}=0.0018-0.0024 \mathrm{eV}, m_{\nu_{2}}=0.0088-0.009 \mathrm{eV}, m_{\nu_{3}}=$ $0.0503 \mathrm{eV}(\mathrm{NH})$ for neutrino masses with a normal hierarchy and where the range arises from different $\tan \beta$ values. Using the $\Delta m_{i j}^{2}$ values given above, a lower limit for the sum of the masses of three neutrino generations $\left(\sum_{i=1,3} m_{v_{i}}\right)$ can be estimated by setting the lightest neutrino mass to zero. 
Cosmological observations can provide an upper bound on the sum. Depending on the model and the data set considered for the analysis, $\sum_{v_{i}}^{\max }$ can range from 0.12 to $1.08 \mathrm{eV}$ at $2 \sigma$ level $[11,16]$.

The current sensitivity of precision LFV measurements already probes certain regions of SUSY parameter space, especially the constrained MSSM (cMSSM or mSUGRA) [17] with light scalar masses and right-handed neutrinos (RHN). The MEG Collaboration reported an upper bound for the branching fraction (BF) of the process $\mu \rightarrow e \gamma: \mathrm{BF}(\mu \rightarrow$ $e \gamma)<4.2 \times 10^{-13}$ at $90 \% \mathrm{CL}$ [18]. Given the limits, sub$\mathrm{TeV}$ cMSSM + RHN models that assume small CKM-like and large PMNS-like Yukawa mixings are excluded, in agreement with the LHC searches. The projected sensitivity of MEG-II should lower the BF limit by another order of magnitude [19]. For $\mu \rightarrow e$ conversion rate (CR) in nuclei, the $\mathrm{Mu} 2 \mathrm{e}$ experiment has a projected sensitivity in the range of $10^{-16}-10^{-17}$. With such a projected sensitivity, Mu2e will be probing the parameter space of MSSM models with nonuniversal Higgs soft masses [20,21].

Even though precision experiments cannot be thought of as a replacement for LHC, they can be complementary. Positive results for sparticle searches at the LHC along with LFV search results would narrow down many beyond-thestandard-model (BSM) theories that are presently compatible with observations. Null results from precision experiments can only constrain models with specific assumptions and so are insufficient to give universal bounds on sparticle masses. Similarly, MSSM + RHN models include PMNS neutrino mixing to explain neutrino oscillations (which is distinct from the CKM-like or PMNS-like Yukawa mixing to be introduced shortly). In addition, the predicted LFV observables might vary by a few orders of magnitude depending on the choice of the RH neutrino mass spectrum for neutrinos (see Fig. 4). ${ }^{1}$

In this paper, we study the predictions of SUSY models with nonuniversal Higgs parameters augmented with three RH neutrino superfields $\hat{N}_{i}^{c}$. Motivated by the normal and inverted mass hierarchies of neutrino masses, we study the threeextra-parameter nonuniversal Higgs model (NUHM3) model [23] with scalar mass relations $m_{0}(1) \simeq m_{0}(2) \neq m_{0}(3)$. We investigate the parameter space where LHC searches, dark matter detection experiments, and LFV observables can be complementary. For each of the different neutrino Yukawa coupling scenarios and mass hierarchies, we take the thirdgeneration neutrino mass to be $\sim 0.05 \mathrm{eV}$ and only accept solutions with $m_{v_{1}}$ and $m_{v_{2}}$ within the limits given in Ref. [11] for both inverse and normal neutrino mass hierarchies.

We also show predictions for the well-motivated NUHM3 model for points that exhibit electroweak naturalness: $\Delta_{\mathrm{EW}} \leqslant$ 30. The naturalness measure $\Delta_{\mathrm{EW}}$ [24] only requires that weak scale contributions to the $Z$-boson mass, $m_{Z}$, should be comparable to or smaller than $m_{Z}$. From the minimization conditions for the MSSM Higgs potential ${ }^{2}$

$$
\frac{m_{Z}^{2}}{2}=\frac{m_{H_{d}}^{2}+\Sigma_{d}^{d}-\left(m_{H_{u}}^{2}+\Sigma_{u}^{u}\right) \tan ^{2} \beta}{\tan ^{2} \beta-1}-\bar{\mu}^{2},
$$

\footnotetext{
${ }^{1}$ For some further references on LFV processes see, e.g., Ref. [22].

${ }^{2}$ We use $\bar{\mu}$ for the SUSY $m u$ term to distinguish it from $\mu$ for muon.
}

electroweak naturalness is defined as $\Delta_{\mathrm{EW}}=\max _{i}\left|C_{i}\right| /$ $\left(m_{Z}^{2} / 2\right)$ where the $C_{i}$ 's represent the various terms on the right-hand side of Eq. (2). Here, $\tan \beta=v_{u} / v_{d}$ is the ratio of Higgs field VEVs with $m_{H_{u}}^{2}$ and $m_{H_{d}}^{2}$ the Higgs soft breaking masses and the $\Sigma_{u}^{u}$ and $\Sigma_{d}^{d}$ terms contain over 40 radiative corrections (for expressions, see Ref. [25]).

One can see that $\Delta_{\mathrm{EW}}<30$ is a conservative condition which accommodates $3.3 \%$ or less fine tuning and allows $\bar{\mu}$ up to $\sim 360 \mathrm{GeV}$. The condition for naturalness puts strong bounds on stop and gluino masses [26]. For various natural MSSM models, and independent of the neutrino sector, upper limits have been calculated as $m_{\tilde{t}_{1}} \lesssim 3.5 \mathrm{TeV}$ and $m_{\tilde{g}} \lesssim 6 \mathrm{TeV}$ [with the exception of the natural anomaly mediated SUSY breaking model (nAMSB) [27] where gluino mass can reach up to $9 \mathrm{TeV}$. Such models will only be partially probed by high luminosity (HL) LHC [28]. For natural SUSY, the NUHM3 allows for heavier first- and second-generation sparticles ranging up to $30-40 \mathrm{TeV}$ without violating the naturalness condition that $\Delta_{\mathrm{EW}}<30$ [29].

In $R$-parity-conserving SUSY models, the lightest SUSY particle (LSP) is expected to be stable. The neutralino LSP has been confronted by direct and indirect dark matter (DM) searches [30]. In this paper, we consider a neutralino LSP with a thermal abundance less than the measured value, $\Omega^{\text {th }} h^{2} \leqslant$ 0.12 . In this case, the neutralino makes up only a fraction of the total dark matter density while the remainder might be composed of axions. In such a scenario, the neutralino abundance can also be augmented by late-decaying relics, such as axinos and saxions [31]. Although direct DM searches probe deeper in SUSY parameter space, indirect searches from FERMI-LAT and other experiments limit the composition of mixed dark matter more strongly [32] since the weakly interacting massive particle (WIMP) indirect detection rates are rescaled by $\xi^{2}=\left(\Omega h^{2} / 0.12\right)^{2}$

In Sec. II, we review the SUSY seesaw mechanism while in Sec. III we discuss possible LFV processes. In Sec. IV, we describe the model parameter space and present the results for NUHM3 + RHN. We summarize projected discovery limits of future LFV experiments in Sec. V.

\section{SEESAW MECHANISM IN MSSM}

The seesaw mechanism is one of the most compelling ways to generate the observed neutrino masses [33]. Implications of supersymmetric models with various seesaw mechanisms have been extensively studied in the literature [34-39]. In supersymmetric models augmented with right-handed neutrinos, loop effects can enhance off-diagonal elements of the slepton mass matrix and hence generate sizable lepton flavor violation effects which results in low energy LFV observables $[34,40]$.

In the supersymmetric type-I seesaw, the MSSM superpotential is of the form

$$
\begin{aligned}
\hat{f}_{\mathrm{MSSM}}= & \bar{\mu} \hat{H}_{u}^{a} \hat{H}_{d a}+\sum_{i, j=1,3}\left[\left(\mathbf{f}_{u}\right)_{i j} \epsilon_{a b} \hat{Q}_{i}^{a} \hat{H}_{u}^{b} \hat{U}_{j}^{c}\right. \\
& \left.+\left(\mathbf{f}_{d}\right)_{i j} \hat{Q}_{i}^{a} \hat{H}_{d a} \hat{D}_{j}^{c}+\left(\mathbf{f}_{e}\right)_{i j} \hat{L}_{i}^{a} \hat{H}_{d a} \hat{E}_{j}^{c}\right] .
\end{aligned}
$$


The MSSM superpotential is augmented by an additional set of terms containing right-handed neutrinos $\hat{N}_{i}^{c}[5]$ :

$$
\hat{f}=\hat{f}_{\mathrm{MSSM}}+\sum_{i, j=1,3}\left[\frac{1}{2}\left(\mathbf{M}_{N}\right)_{i j} \hat{N}_{i}^{c} \hat{N}_{j}^{c}+\left(\mathbf{f}_{v}\right)_{i j} \epsilon_{a b} \hat{L}_{i}^{a} \hat{H}_{u}^{b} \hat{N}_{j}^{c}\right],
$$

where $\mathbf{M}_{N}$ is the Majorana mass matrix for the heavy righthanded neutrinos. We work in a basis where the $\mathbf{f}_{d}$ and $\mathbf{f}_{e}$ are diagonal. Additionally, $\mathbf{M}_{N}$ is taken to be diagonal at the grand unified theory (GUT) scale.

Since the entirety of neutrino mixing data is insufficient to fix all the elements of $\mathbf{f}_{v}$, it is common to assume in addition some GUT-motivated Ansätze which can span the realm of possibilities [20]. Three common scenarios include the following:

(i) scenario No. 1: $\left(\mathbf{f}_{v}\right)_{i j}=\left(\mathbf{f}_{u}\right)_{i j}$ small mixing (CKM like),

(ii) scenario No. 2: $\left(\mathbf{f}_{v}\right)_{i j}=\left(\mathbf{f}_{u}\right)_{i i}^{\text {diag }} \mathbf{U}_{i j}^{\text {PMNS }}$ large mixing (PMNS like), and

(iii) scenario No. 3: $\left(\mathbf{f}_{v}\right)_{i j}=3 \times\left(\mathbf{f}_{u}\right)_{i j}$,

where the conditions are imposed at the GUT scale $m_{\mathrm{GUT}}$ [20]. These relations are shown more explicitly in Appendix A along with numerical realizations. One simple GUT-motivated scenario is where Higgs superfields reside in 10-plets; in that case, then scenario No. 1 is the trivial choice, whereas for the fields that reside in 126-plets, then scenario No. 3 should be considered for models with CKM-like neutrino couplings. For a given light neutrino spectrum, in scenario No. 3 Majorana neutrinos should be chosen a factor of $\sim 3^{2}=9$ heavier compared to the scenario No. 1. Hence, LFV observables are expected to be diminished by a factor of $\propto \log 9$ and enhanced by a factor of 9 due to the squared neutrino coupling in calculation: $\left(3 \times f_{v}\right)^{2}$. For simplicity, we only show results for scenario No. 1 (small mixing, CKM like) and scenario No. 2 (large mixing, PMNS like). While the above GUT scenarios are likely to live in the swampland [41] of string inconsistent models (due to the presence of large Higgs multiplets [42]), the large and small mixing cases can also be present in stringderived models (such as heterotic models compactified on certain orbifolds [43]) which only include the MSSM + RHN fields in the low-energy spectrum, and where GUT multiplets arise from local grand unification [44] present at orbifold fixed points.

Above the highest seesaw scale set by the heaviest Majorana neutrino, denoted by $N_{3},{ }^{3}$ the light neutrino mass matrix can be written as

$$
\left(\mathbf{m}_{v}\right)_{i l}=-(v \cdot \sin \beta)^{2}\left(\mathbf{f}_{v}^{T}\right)_{i j}\left(\mathbf{M}_{N}^{-1}\right)_{j k}\left(\mathbf{f}_{v}\right)_{k l},
$$

where $v \equiv \sqrt{v_{u}^{2}+v_{d}^{2}} \simeq 174 \mathrm{GeV}$ is the combined vacuum expectation value of the Higgs bosons in the MSSM and where $\beta=\arctan \left(v_{u} / v_{d}\right)$. The magnitudes of the neutrino Yukawa couplings $\left(\mathbf{f}_{v}\right)_{i j}$ and Majorana mass matrix $\left(\mathbf{M}_{N}\right)_{j k}$ in Eq. (5) are functions of the energy scale $Q$. When the heaviest Majorana neutrino is integrated out, an effective dimension-5

\footnotetext{
${ }^{3}$ We consider a model with three nondegenerate right-handed neutrinos.
}

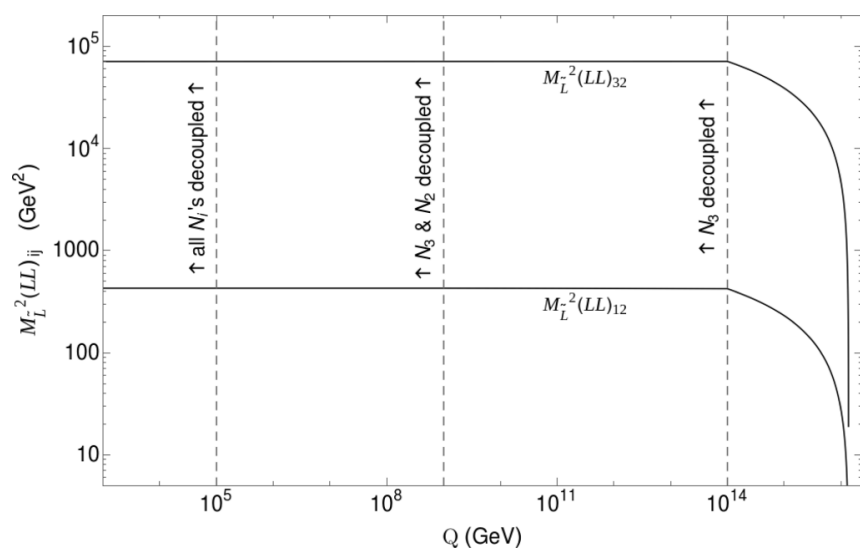

FIG. 1. Evolution of the off-diagonal slepton mass matrix terms $m_{\widetilde{L}}^{2}(L L)_{12}$ and $m_{\widetilde{L}}^{2}(L L)_{32}$ with the energy scale $Q$ from $m_{\mathrm{GUT}}$ to $m_{Z}$ with $M_{N_{1,2,3}}=10^{5,9,14} \mathrm{GeV}$ (denoted by vertical dashed lines).

neutrino mass operator is generated :

$$
\hat{f} \ni \frac{1}{2}(\kappa)_{i l} \epsilon_{a b} \hat{L}_{i}^{a} \hat{H}_{u}^{b} \epsilon_{d f} \hat{L}_{l}^{d} \hat{H}_{u}^{f},
$$

where the coefficient $\kappa$ of the operator can be determined by the matching condition at the decoupling scales [45]:

$$
\left.(\kappa)_{i l}\right|_{M_{N_{k}}^{-}}=\left.(\kappa)_{i l}\right|_{M_{N_{k}}^{+}}+\left.\left(\mathbf{f}_{v}^{T}\right)_{i k}\left(M_{N_{k}}^{-1}\right)\left(\mathbf{f}_{v}\right)_{k l}\right|_{M_{N_{k}}^{+}},
$$

where $k=1,2,3$ and where $M_{N_{k}}^{+}\left(M_{N_{k}}^{-}\right)$denotes the value as the scale of decoupling of the $k$ th generation RHN $M_{N_{k}}$ is approached from above (below). At $Q=M_{N_{k}}, \hat{N}_{k}$ is integrated out, hence, heavy neutrinos decouple from the theory. For $Q>M_{N_{3}}, \kappa=0$ and RG evolution only governs $\mathbf{f}_{v}$ and $M_{N}$. After the heaviest neutrino is decoupled, the neutrino mass matrix becomes

$$
\left(\mathbf{m}_{v}\right)_{i l}=-\frac{1}{2}(v \cdot \sin \beta)^{2}\left[\kappa_{i l}+\left(\mathbf{f}_{v}^{T}\right)_{i j}\left(\mathbf{M}_{N}^{-1}\right)_{j k}\left(\mathbf{f}_{v}\right)_{k l}\right] .
$$

After all the RH neutrinos are decoupled, the RGEs only govern $\kappa$ from the scale of the lightest heavy neutrino mass $M_{N_{1}}$, all the way down to the electroweak breaking scale. For $Q<M_{N_{1}}$, the neutrino mass matrix is given by

$$
\left(\mathbf{m}_{v}\right)_{i l}=-\frac{1}{2}(v \cdot \sin \beta)^{2} \kappa_{i l},
$$

and physical light neutrino masses can be obtained by diagonalizing $\left(\mathbf{m}_{v}\right)_{i l}$.

In Fig. 1, we show the evolution of two off-diagonal slepton mass matrix terms with the energy scale $Q$ from $m_{\mathrm{GUT}}$ to the weak scale using matching conditions at the thresholds and the small mixing (CKM-like) neutrino Yukawa couplings at $m_{\mathrm{GUT}}$. The RH neutrino masses are shown by the dashed lines to be $M_{N_{1,2,3}}=10^{5,9,14} \mathrm{GeV}$. During the running, the off-diagonal terms receive contributions proportional to $Y_{t}^{2} V_{31}^{\mathrm{CKM}} V_{32}^{\mathrm{CKM}}$ for $Q>M_{N_{3}}$, to $Y_{c}^{2} V_{21}^{\mathrm{CKM}} V_{22}^{\mathrm{CKM}}$ for $M_{N_{3}}>Q>M_{N_{2}}$ and to $Y_{u}^{2} V_{11}^{\mathrm{CKM}} V_{12}^{\mathrm{CKM}}$ for $M_{N_{2}}>Q>M_{N_{1}}$ for the CKM-like mixing as shown in the figure. Since the third-generation up-type Yukawa coupling dominates over first and second generations, the main contribution from the RGEs is during $Q>M_{N_{3}}$. For $Q<M_{N_{3}}$, the renormalization group equation (RGE) running effects on LFV observables are highly suppressed due to the smallness of up and charm quark 

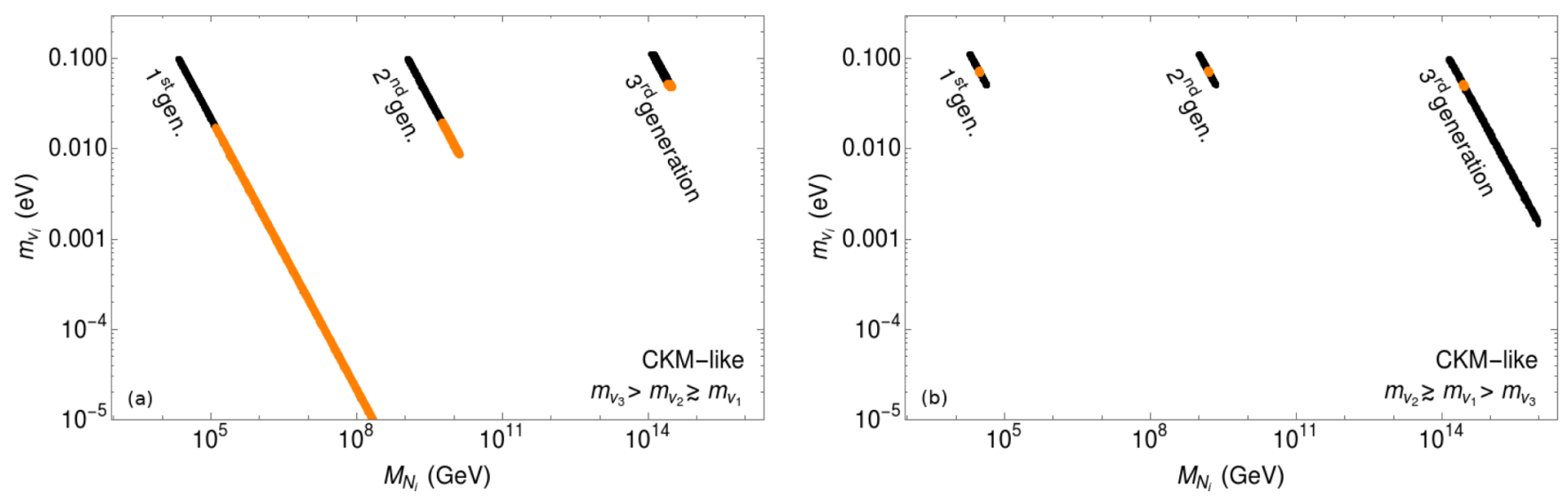

FIG. 2. Dependence of light neutrino masses on heavy neutrino masses for normal hierarchy (a) and inverse hierarchy (b). The parameter space compatible with $m_{v_{3}} \simeq 0.05 \mathrm{eV}$ are colored in orange.

Yukawa couplings. Using the method described above, the computed light neutrino masses $\left(m_{v_{i}}\right)$ as a function of heavy neutrino masses $\left(M_{N_{i}}\right)$ are shown in Fig. 2 for both normal $(\mathrm{NH})$ and inverted $(\mathrm{IH})$ light neutrino mass hierarchies using the small CKM-like neutrino mixings (scenario No. 1). The parameter space of neutrinos studied in this paper is shown in orange. For values $m_{v_{3}}<10^{-3} \mathrm{eV}, M_{N_{3}}$ must be close to $m_{\mathrm{GUT}}$ so we show $M_{N_{3}}$ up to $10^{16} \mathrm{GeV}$ for the inverted hierarchy case. The summed neutrino masses are bounded above by $\max \left(\sum v_{i}\right)=0.31(0.32) \mathrm{eV}$ for normal (inverted) hierarchy from the analysis of the $\Lambda \mathrm{CDM}+\sum+A_{\text {lens }}$ model using the Planck TT,TE,EE $+\tau_{\mathrm{HFI}}+\mathrm{BAO}$ data set [11].

The sizes of the off-diagonal elements in the slepton mass matrix are mostly sensitive to $\log \left(m_{\mathrm{GUT}} / M_{N_{3}}\right)$. As further discussed in the next section, LFV rates strongly depend on the mass of the third-generation heavy neutrino, so the rates are not expected to show a difference for a different set of neutrino mass choice with normal hierarchy since $M_{N_{3}}$ can only take values within a small range. With $\mathrm{IH}$, it is always possible to suppress SUSY enhanced LFV observables by taking $M_{N_{3}} \sim m_{\text {GUT }}$ as seen in Fig. 1 . We adopt $m_{v_{3}} \simeq 0.05 \mathrm{eV}$ as the common third-generation neutrino mass.

\section{LEPTON FLAVOR VIOLATION AND OBSERVABLES}

In supersymmetry, nondiagonal mass matrix elements in the slepton mass matrix can be the source of LFV processes [40]. For example, in the mass insertion (MI) method with leading-logarithm approximation, the branching fractions for processes $l_{i} \rightarrow l_{j} \gamma$ can be approximated as

$$
\mathrm{BF}\left(l_{i} \rightarrow l_{j} \gamma\right) \simeq\left|\left(m_{\widetilde{L}}^{2}\right)(L L)_{i j}\right|^{2} \frac{\alpha^{3} \tan ^{2} \beta}{G_{F}^{2} m_{S}^{8}},
$$

where $\alpha$ is the fine-structure constant, $G_{F}$ is the Fermi constant, and $m_{S}$ defines the mass scale of the SUSY particles [40]. In the MI technique, one defines the $6 \times 6$ slepton mass matrix as

$$
m_{\widetilde{L}}^{2}=\left(\begin{array}{ll}
m_{\widetilde{L}}^{2}(L L)_{i j} & m_{\widetilde{L}}^{2}(L R)_{i j} \\
m_{\widetilde{L}}^{2}(R L)_{i j} & m_{\widetilde{L}}^{2}(R R)_{i j}
\end{array}\right),
$$

where $L L, L R, R L$, and $R R$ are $3 \times 3$ entries defined based on the chirality label of the sfermions.

In the MSSM (with no RHNs), off-diagonal elements of the slepton mass matrix are small enough to not give rise to significant flavor-violating rates. Hence, flavor-changing processes such as $\mu \rightarrow e \gamma$ are suppressed. When heavy righthanded neutrinos are introduced into the model, RG running can give rise to large values for the mass matrix elements. In SO(10) SUSY models, the dominant contribution arising from the neutrino sector is proportional to the squared top-quark Yukawa coupling $Y_{t}^{2}$. Hence, the size of the elements of the slepton mass matrix associated with LFV processes depends mostly on the running from $m_{\mathrm{GUT}}$ to $M_{N_{3}}$.

\section{A. $\mu \rightarrow e \gamma$}

In supersymmetry, flavor-changing processes are induced by one-loop diagrams with the exchange of gauginos and sleptons (see Fig. 3 for neutralino and chargino diagrams). The leading-logarithm approximation for the slepton mass matrix element that induces the process $\mu \rightarrow e \gamma$ reads as

$$
\left(m_{\widetilde{L}}^{2}\right)_{12} \simeq-\frac{1}{8 \pi^{2}}\left(2 m_{0}^{2}+m_{H_{u}}^{2}+A_{0}^{2}\right) \sum_{k}\left(\mathbf{f}_{v}^{\mathbf{T}}\right)_{1 k}\left(\mathbf{f}_{v}{ }^{*}\right)_{k 2} \log \frac{m_{\mathrm{GUT}}}{M_{N_{k}}} .
$$

For low values of $m_{1 / 2}$, the approximation holds well but for values $m_{1 / 2} \simeq 1 \mathrm{TeV}$, the branching fractions can be up to a factor of 10 different than estimates from RGE running [46]. If the hierarchy of neutrino couplings is similar to that of the up-type in the standard model, where the third generation dominates, then the largest contribution is from the $k=3$ term in the summation. $\operatorname{BF}(\mu \rightarrow e \gamma)$ can be calculated by substituting the $m_{\widetilde{L}}^{2}$ term in Eq. (12) into the expression in (10) for a good approximation for a light/pre-LHC SUSY mass spectrum.

In the mass insertion technique, the branching fraction for the process $\mu \rightarrow e \gamma$ can be calculated using

$$
\mathrm{BF}(\mu \rightarrow e \gamma)=\frac{48 \pi^{3} \alpha}{G_{F}^{2}}\left(\left|A_{L}^{\mu e}\right|^{2}+\left|A_{R}^{\mu e}\right|^{2}\right),
$$



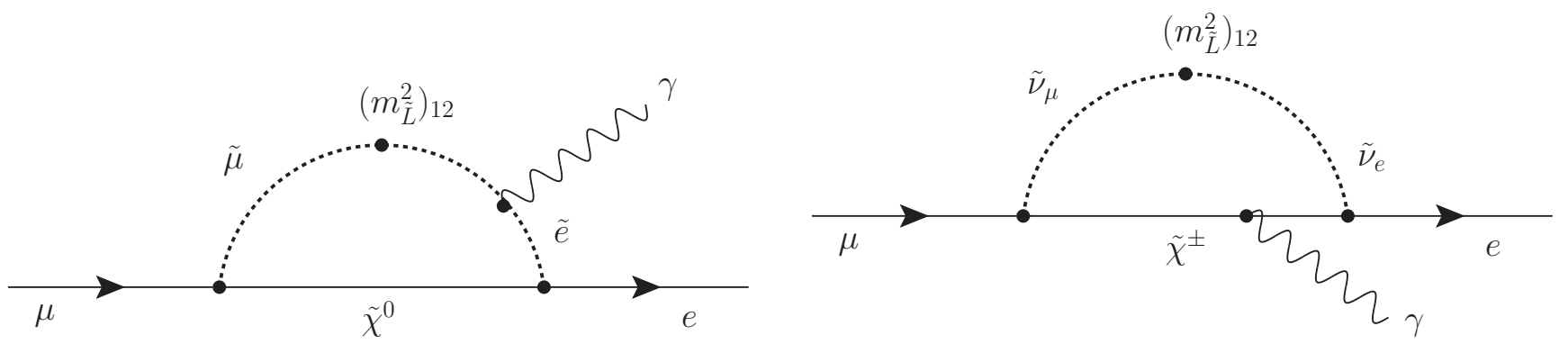

FIG. 3. Examples of Feynman diagrams contributing to $\mu \rightarrow e \gamma$ in MSSM + RHN models.

where the amplitudes $A_{L}$ 's and $A_{R}$ 's are given in Ref. [34]. The MEG collaboration reported the current bound for the process as $\mathrm{BF}(\mu \rightarrow e \gamma)<4.2 \times 10^{-13}$ at $90 \% \mathrm{CL}$ [18]. The expected sensitivity of the MEG-II experiment is $6 \times 10^{-14}$ for three years of data taking [47].

\section{B. $\tau \rightarrow \mu \gamma$}

In a similar way to the leading-logarithm approximation for $\mu \rightarrow e \gamma$ decay, $\mathrm{BF}(\tau \rightarrow \mu \gamma)$ can be approximated as

$\left(m_{\widetilde{L}}^{2}\right)_{32} \simeq-\frac{1}{8 \pi^{2}}\left(2 m_{0}^{2}+m_{H_{u}}^{2}+A_{0}^{2}\right) \sum_{k}\left(\mathbf{f}_{v}^{\mathbf{T}}\right)_{3 k}\left(\mathbf{f}_{v}{ }^{*}\right)_{k 2} \log \frac{m_{\mathrm{GUT}}}{M_{N_{k}}}$,

where the dominant contribution comes from the term with $\left(\mathbf{f}_{v}^{\mathbf{T}}\right)_{33}\left(\mathbf{f}_{v}{ }^{*}\right)_{32} \propto Y_{t}^{2} V_{t b} V_{t s}$ in models with CKM-like neutrino Yukawa mixings and from the term $\propto Y_{t}^{2} U_{\mu 3} U_{\tau 3}$ in models with PMNS-like mixings [20]. The most stringent current constraint comes from $\mathrm{BaBar}$ collaboration $\mathrm{BF}(\tau \rightarrow \mu \gamma)<$ $4.4 \times 10^{-8}$ at $90 \%$ CL [48]; the Belle-II experiment expects to gain a factor of 10 improvement [49].

\section{C. $\mu \rightarrow 3 e$}

In SUSY, the decay $\mu \rightarrow 3 e$ gets contributions from $\gamma$-, $Z$-, Higgs-penguin and box diagrams where the $\gamma$ penguin is the dominant one [50]. The leading $\gamma$-penguin approximation gives the relation

$$
\frac{\mathrm{BF}(\mu \rightarrow 3 e)}{\mathrm{BF}(\mu \rightarrow e \gamma)}=\frac{\alpha}{3 \pi}\left(\log \frac{m_{\mu}^{2}}{m_{e}^{2}}-\frac{11}{4}\right)
$$

The current experimental lower bound on $\mathrm{BF}(\mu \rightarrow e e e)$ is from the SINDRUM experiment: $10^{-12}$ [51]. The proposed Mu3e experiment has a projected sensitivity of $5.2 \times 10^{-15}$ [52] up to $\sim 10^{-16}$ at phase II.

$$
\text { D. } \mu N \rightarrow e N
$$

The $\mu \rightarrow e$ conversion rate (CR) inside a nucleus $N$ depends on the neutron and proton densities. The rate is larger with increasing $Z$ (atomic number) up to $Z \sim 30$ and decreasing for $Z \gtrsim 60$ [53]. The Mu2e experiment is designed to look for $\mu N \rightarrow e N$ conversion with a sensitivity of conversion rate $\mathrm{CR}(\mu+A l \rightarrow e+A l) \sim 2.4 \times 10^{-17}$ in aluminum [54]. The sensitivity is improved by replacing aluminum targets with titanium ones.

\section{RESULTS FOR SUSY MODELS WITH NONUNIVERSAL SCALAR MASSES}

The cMSSM + RHN model parameter set has been well investigated in the literature [20,35,37,38]. Also, the NUHM1,2 + RHN models were previously studied for specific nonuniversal scenarios with the following GUT-scale mass relations: NUHM1 $\left(m_{H_{u}}^{2}=m_{H_{d}}^{2} \neq m_{0}^{2}\right)$ in Ref. [20], NUHM2 $\left(m_{H_{u}}^{2} \neq m_{H_{d}}^{2} \neq m_{0}^{2}\right)$, and NUGM (nonuniversal gaugino masses with $\left.M_{1}: M_{2}: M_{3}=1 / 2: 3 / 2: 1\right)$ in Ref. [21]. In these papers, the authors fixed the RH neutrino masses at $m_{\mathrm{GUT}}$. Here, we adopt the three-extra-parameter nonuniversal Higgs mass SUSY model NUHM3 wherein the first and second generations of matter scalars are split from the third $\left[m_{H_{u}}^{2} \neq m_{H_{d}}^{2} \neq m_{0}^{2}(3) \neq m_{0}^{2}(1,2)\right]$. The NUHM3 model is well motivated in that it allows for natural SUSY with $\Delta_{\text {EW }}<30$ (and the required small $\bar{\mu}$ parameter) while also allowing for third-generation nonuniversality as is expected in many stringy constructs $[29,55]$.

We scan over the following parameter space:

\begin{tabular}{lcc}
\hline MSSM parameters & & sneutrino parameters \\
\hline$m_{0}(3)$ & $: 0-20 \mathrm{TeV}$ & $m_{\tilde{v}_{3}}=m_{0}(3)$ \\
$m_{0}(1,2)$ & $: 0-20 \mathrm{TeV}$ & $m_{\tilde{v}_{1,2}}=m_{0}(1,2)$ \\
$m_{1 / 2}$ & $A_{v}=A_{0}$ \\
$A_{0}$ & $: 0-4 \mathrm{TeV}$ & \\
$\bar{\mu}$ & $:(-4-4) m_{0}(3)$ & \\
$m_{A}$ & $: 0-10 \mathrm{TeV}$ & \\
$\tan \beta$ & $: 3-60$ & $m_{v_{3}} \simeq 0.05 \mathrm{eV}$ (weak scale) \\
\hline
\end{tabular}

The first four SUSY breaking masses are input at the GUT scale; $\bar{\mu}$ and $m_{A}$ are input at the weak scale. Weak scale values of $\bar{\mu}$ and $m_{A}$ are preferred instead of $m_{H_{u}}$ and $m_{H_{d}}$ at GUT scale for a better statistical sampling of $\bar{\mu} \leqslant 1 \mathrm{TeV}$. We also assume $\bar{\mu}$ to be positive and real. The lower-end limits of SUSY breaking terms and $\bar{\mu}$ are in tension with LHC and LEP2 searches. More precisely, $m_{\tilde{g}}>2.25 \mathrm{TeV}$ and $m_{\tilde{x}_{1}^{ \pm}}>103.5$ $\mathrm{GeV}$ require $m_{1 / 2} \gtrsim 1 \mathrm{TeV}$ and $\bar{\mu} \gtrsim 100 \mathrm{GeV}$, respectively. We pay some special attention to natural SUSY solutions with $\Delta_{\text {EW }}<30$ since these generate a weak scale of $m_{\text {weak }} \sim 100$ $\mathrm{GeV}$ without requiring any large fine tunings in Eq. (2).

Only the solutions that give the neutrino mass spectrum of interest $\left(m_{v_{3}}=0.05 \pm 0.0025 \mathrm{eV}\right.$ and $m_{v_{1,2}}$ determined by $\Delta m_{i j}^{2}$ given in Ref. [11]) after the RGE running are accepted. Results are shown in two different categories based on the 

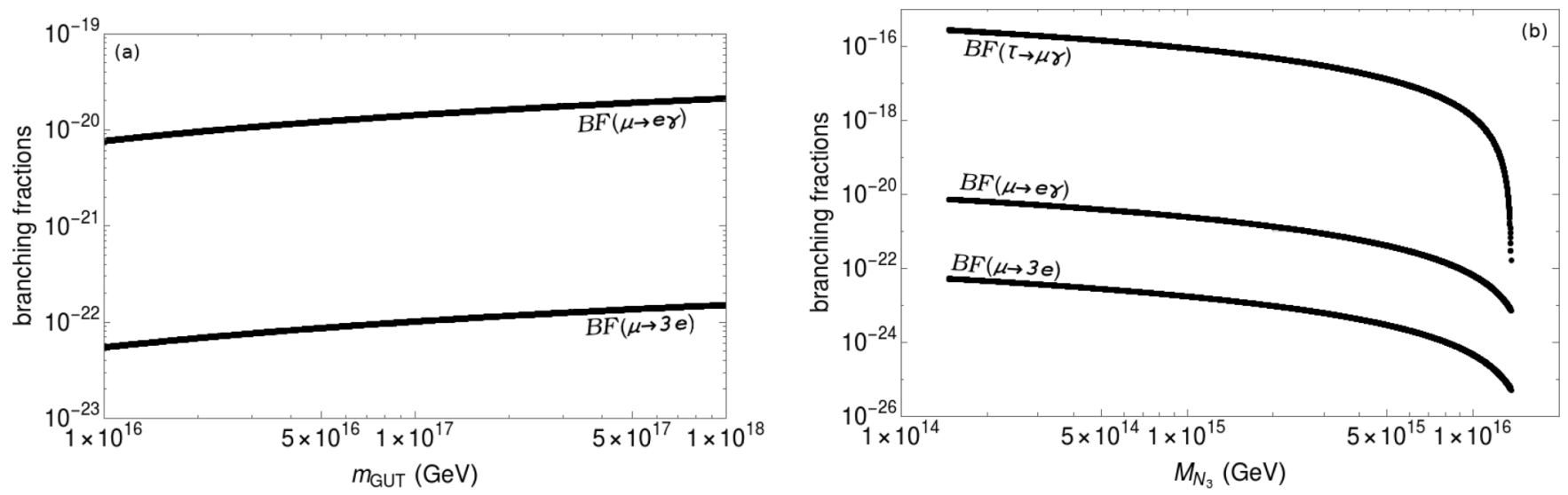

FIG. 4. Dependence of $\mathrm{BF}(\mu \rightarrow e \gamma)$ (a) to $m_{\mathrm{GUT}}$ with fixed $M_{N_{1,2,3}} \equiv 10^{6,10,14} \mathrm{GeV}$ and (b) to $M_{N_{3}}$ for inverse hierarchical light neutrinos.

sneutrino mass ordering at the weak scale:

$$
\begin{aligned}
& m_{\tilde{v}_{3}}>m_{\tilde{v}_{2}} \simeq m_{\tilde{v}_{1}} \rightarrow \text { normal sneutrino ordering (NSO), } \\
& m_{\tilde{v}_{3}}<m_{\tilde{v}_{2}} \simeq m_{\tilde{v}_{1}} \rightarrow \text { inverted sneutrino ordering (ISO). }
\end{aligned}
$$

Since first- and second-generation masses can drastically increase during RGE running for heavy enough gluinos, imposing a hierarchy on scalars $\left[m_{0}(1,2,3)\right.$ 's] at the GUT scale does not guarantee that one generates the same hierarchy at the weak scale. Furthermore, LFV processes such as $\mu \rightarrow e \gamma$ and $\mu \rightarrow e e e$ are directly related to the sneutrino mass hierarchy rather than the scalar mass hierarchy at the weak scale. In the rest of the paper, we use the terms NSO and ISO for the weak scale ordering of sneutrinos.

We adopt a top-down approach: all the parameters are evolved from $m_{\mathrm{GUT}}$ to $m_{\text {weak }}$ with the RH neutrinos decoupled one by one using the methodology of Antusch et al. [45]. The down-type Yukawa, lepton Yukawa, and heavy neutrino mass matrices are all taken to be diagonal at $m_{\mathrm{GUT}}$. SUSY spectra are calculated by using ISAJET [56] with neutrinorelated RGEs calculated using a modified version of the subroutine ISABRS that comes within the ISAJET package. After RG running from $m_{\mathrm{GUT}}$ to $m_{\text {weak }}$, the mass matrices $m_{\tilde{L}}^{2}, m_{\tilde{v}}^{2}, m_{\tilde{\chi}^{0}}^{2}$, and $m_{\tilde{\chi}^{ \pm}}^{2}$ are calculated at the weak scale. We accept solutions that give the observed neutrino oscillations and with a dark matter abundance less than the measured result $\Omega h^{2} \leqslant 0.12$ (assuming that the remainder of the dark matter might be composed of axions). Light neutrino masses are calculated by diagonalizing the coupling of the dim-5 operator $\kappa$. We use the package SUSEFLAV [57] to calculate $\mathrm{BF}(\mu \rightarrow e \gamma), \mathrm{BF}(\tau \rightarrow \mu \gamma)$, and $\mathrm{BF}(\mu \rightarrow e e e)$ by feeding in the low-energy spectrum after RG running of ISAJET. SUSEFLAV calculates flavor-changing observables using MIs. To calculate the $\mathrm{CR}(\mu N \rightarrow e N)$ for aluminum and titanium nuclei, we adopt the values for the effective nuclear charge $Z_{\mathrm{eff}}^{\mathrm{Al}}=11.6$ and the form factor $F(q)=0.64$ for ${ }_{13}^{27} \mathrm{Al}$ and $Z_{\text {eff }}^{T i} \simeq 17.6$ with $F(q)=0.52$ for ${ }_{22}^{48} \mathrm{Ti}[53]$.

The main contribution to off-diagonal elements of the slepton mass matrix arises during the evolution from $Q=$ $m_{\mathrm{GUT}} \rightarrow M_{N_{3}}$, since the $\mathrm{BF}(\mu \rightarrow e \gamma) \propto\left(f_{v}\right)_{3,3}^{2}$, so the mass of the third-generation heavy neutrino plays a crucial role for the LFV processes. Our choice of $M_{N_{3}}$ generates nearly maximal LFV effects for both sneutrino hierarchies NSO and ISO.

We only show solutions with:

(i) neutralino LSP,

(ii) radiatively broken electroweak symmetry,

(iii) the lightest chargino mass greater than $103.5 \mathrm{GeV}$ (LEP2 limit) [58],

(iv) Higgs mass $m_{h}=125 \pm 2 \mathrm{GeV}$,

(v) $1.12 \times 10^{-9}<\mathrm{BF}\left(B_{S} \rightarrow \mu^{+} \mu^{-}\right)<4.48 \times 10^{-9}$ [59],

(vi) $2.79 \times 10^{-4}<\mathrm{BF}(b \rightarrow s \gamma)<4.63 \times 10^{-4}$ [59].

In Fig. 4(a), we show the effect of taking $m_{\mathrm{GUT}}$ larger than the SUSY unification scale on the $\mathrm{BF}(\mu \rightarrow e \gamma)$ while keeping $M_{N_{3}}$ fixed at $10^{14} \mathrm{GeV}$. It is possible to assume $m_{\mathrm{GUT}}$ close to $M_{\text {string }}$ which is considered to be slightly higher than the gauge coupling unification scale due to string loop effects [60]. Taking $m_{\mathrm{GUT}}$ larger than the gauge coupling unification scale increases the rates for LFV processes since $M_{N_{3}}$ is fixed. The effect of increasing $m_{\mathrm{GUT}}$ by two orders of magnitude increases LFV effects by a factor of 2 which is what we expect from the leading-logarithm approximation (12). In the remainder of our analysis, we take $m_{\mathrm{GUT}}$ to be equal to the gauge coupling unification scale: $\sim 2.4 \times 10^{16} \mathrm{GeV}$.

In Fig. 4(b), we show how sensitive LFV processes are to our choice of neutrino mass spectrum in the IH case. In the $\mathrm{IH}$ case, $M_{N_{3}}$ can take values from $\sim 10^{14} \mathrm{GeV}$ (from cosmological bound on sum of neutrino masses $)$ to $\sim m_{\mathrm{GUT}}(0.001<$ $\left.m_{v_{3}} / \mathrm{eV}<0.1\right)$ whereas first and second generations are more constrained. As seen in the figure, the branching fractions drop significantly for $M_{N_{3}} \gtrsim 5 \times 10^{15} \mathrm{GeV}$ (corresponding to $m_{\nu_{3}}<0.002 \mathrm{eV}$ ) since SUSY contributions to LFV approach zero for $M_{N_{3}} \sim m_{\mathrm{GUT}}$. Hence, it is hard to rule out models with the IH unless there is a lower bound on $m_{v_{3}}$ from observations.

\section{A. Results for branching fractions}

The branching fractions for leptonic decays $l_{i} \rightarrow l_{j} \gamma$ are inversely proportional to $m_{\text {SUSY }}^{8}$ in the leading-logarithm approximation where $m_{\mathrm{SUSY}}$ is the sparticle mass scale [46]. The correlation between $m_{\mathrm{SUSY}}$ and $\mathrm{BF}(\mu \rightarrow e \gamma)$ is shown in Fig. 5(a). The $\mathrm{BF}(\mu \rightarrow e \gamma)$ is decreasing with increasing $m_{\text {SUSY }}$ as expected $[40,61]$. Here, we take $m_{\text {SUSY }}$ as the 

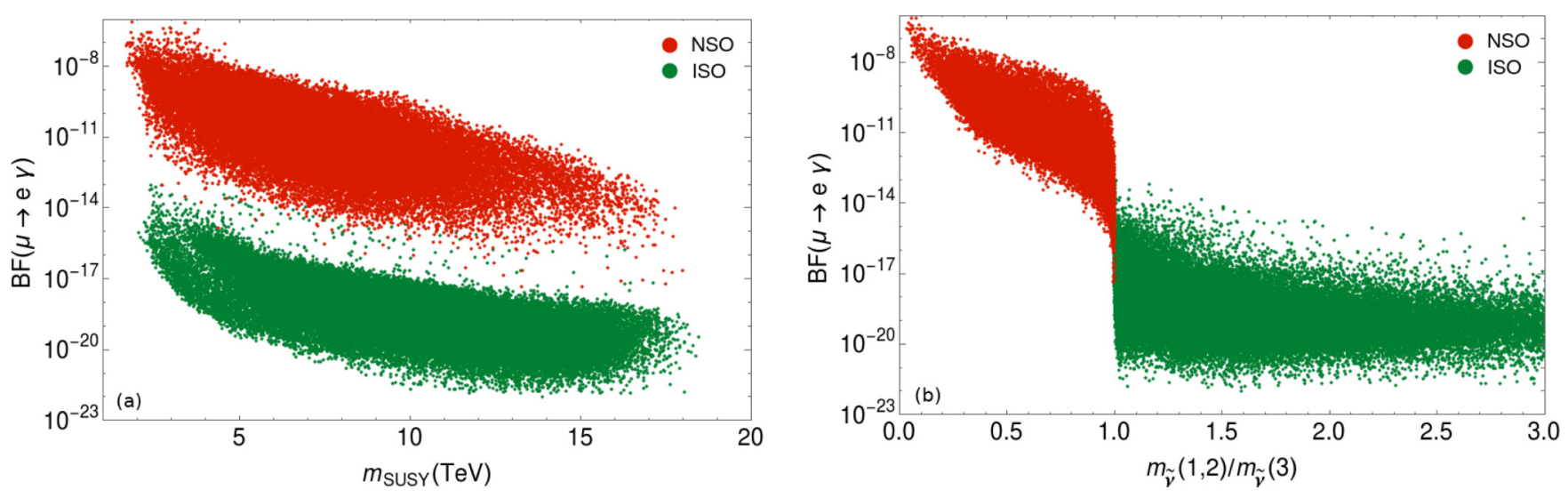

FIG. 5. $\mathrm{BF}(\mu \rightarrow e \gamma)$ vs $m_{\mathrm{SUSY}}$ (a) and vs ratio of the first- and second-generation to third-generation sneutrino mass $m_{\tilde{v}_{1,2}} / m_{\tilde{v}_{3}}(\mathrm{~b})$, both with CKM-like neutrino mixing. Red: NSO and green: ISO.

arithmetic mean of the sfermion masses and the gluino mass. We constrain the parameter space to be within the HE-LHC sparticle mass reach [26]: $m_{\tilde{g}} \lesssim 6 \mathrm{TeV}$. The main feature of the NUHM3 model investigated here arises from the ratio of firstand second-generation to third-generation sneutrino masses as shown in Fig. 5(b). Due to cancellations and decouplings within the amplitudes of chargino and neutralino loop diagrams, LFV observables are highly suppressed for sparticles with the ISO. Hence, $\mu \rightarrow e \gamma$ decays are highly suppressed for $m(3)<m(2) \simeq m(1)$. This behavior is expanded upon in Appendix B.

\section{Scenario No. 1: Small CKM-like mixing $\left(\mathrm{f}_{v}\right)_{i j}=\left(\mathrm{f}_{u}\right)_{i j}$}

Our results for LFV observables are shown in Fig. 6 where we show branching fractions for the LFV decays $\mu \rightarrow e \gamma$, $\tau \rightarrow \mu \gamma$, and $\mu \rightarrow$ eee as a function of $m_{\tilde{g}}$ for sneutrino masses with the NSO (left) and with the ISO (right). Current limits are shown by the solid horizontal lines while projected reaches of future LFV experiments MEG-II, Belle-II, and Mu3e are shown by dashed lines.

For the small (CKM-like) mixing case, the transition from normal to inverted sneutrino mass ordering shows a sharp decrease for the branching fractions $\mu \rightarrow e \gamma$ and $\mu \rightarrow e e e$ when the dominant contribution to the amplitude $A_{L, R}^{\mu e, \mu e e e}$ from the chargino loop $A_{L, R}^{C}$ changes sign which strongly depends on the hierarchy of sneutrino masses. The transition is smoother in the large mixing (PMNS-like) case since the cancellations between $A_{L, R}^{i, j}$ are not as strong as in the small mixing case.

From Fig. 6, we see that the MEG2013 result has ruled out the NSO (left) of sfermions for the majority of the parameter space. This includes the bulk of natural SUSY points (yellow). Surviving points have highly degenerate mass spectra $m(3) \simeq$ $m(2) \simeq m(1)$ which might be expected from NUHM2 boundary conditions. Constraints from the other search channels $\tau \rightarrow \mu \gamma$ and $\mu \rightarrow e e e$ are less stringent.

The ISO case (right) in all channels predicts very mild flavor violation which may not be within the reach of projected sensitivities. The ISO with $m_{0}(3) \ll m_{0}(1,2)$ is actually favored by SUSY string landscape predictions which prefer soft SUSY breaking masses as large as possible subject to appropriate electroweak symmetry breaking (EWSB) [29]. In this case, all natural SUSY points lie below projected limits. Although $\mathrm{BF}(\tau \rightarrow e \gamma)$ is large due to large CKM mixing element $\left|V_{t b}\right| \sim \mathcal{O}(1)$, the Belle-II sensitivity is still higher than the predicted $\tau \rightarrow e \gamma$ decay rates.

The predictions for natural SUSY (yellow points) with a NSO (left) are all above the existing MEG2013 limits. This is mainly because from naturalness $m_{0}(3)$ cannot exceed $\sim 5-10 \mathrm{TeV}$ lest contributions to the weak scale become large and the model becomes unnatural. For the NSO, the first- and second-generation sneutrino masses are necessarily lighter than the third generation, so this acts to enhance the LFV rates.

For the (string preferred) ISO scenario [Fig. 6(right) yellow points], natural SUSY predicts much lower rates for LFV branching fractions because now first- and second-generation sleptons can range up to the scan upper limits (as large as $20 \mathrm{TeV}$ ) with little effect on naturalness while thirdgeneration scalars, which are more strongly related to naturalness, remain in the several $\mathrm{TeV}$ range. Hence, points with $m_{0}(1,2) \simeq 20 \mathrm{TeV}$ (upper scan limit) set the lower bound for LFV processes for ISO for natural SUSY. As the gluino mass gets larger, the yellow points tend to populate lower BF values mainly because naturalness can be preserved with smaller $\tan \beta, \tan \beta<20$ as $m_{1 / 2}$ gets larger. The overall conclusion from the ISO plots of Fig. 6 is that the stringy preferred [29,55] natural SUSY models tend to predict LFV decay rates well below present and even future experimental search limits.

\section{Scenario No. 2: Large PMNS-like mixing $\left(\mathbf{f}_{v}\right)_{i j}=\left(\mathbf{f}_{u}\right)_{i i}^{\text {diag }} \mathbf{U}_{i j}^{\text {PMNS }}$}

In this section, we present predictions for LFV decays from the NUHM3 model but with scenario No. 2 large PMNS-like mixing of neutrino couplings. The cMSSM with PMNS-like mixing is already under tension from MEG results whereas the NUHM1 model with $\tan \beta<20$ survives [20,21].

Predictions from the NUHM3 model for the LFV branching fractions $\mathrm{BF}(\mu \rightarrow e \gamma), \mathrm{BF}(\tau \rightarrow \mu \gamma)$, and $\mathrm{BF}(\mu \rightarrow e e e)$ are shown in Fig. 7 for the large PMNS-like mixing case for the NSO (left) and ISO (right). The large PMNS-like mixing predicts higher LFV rates so more of the parameter space is 

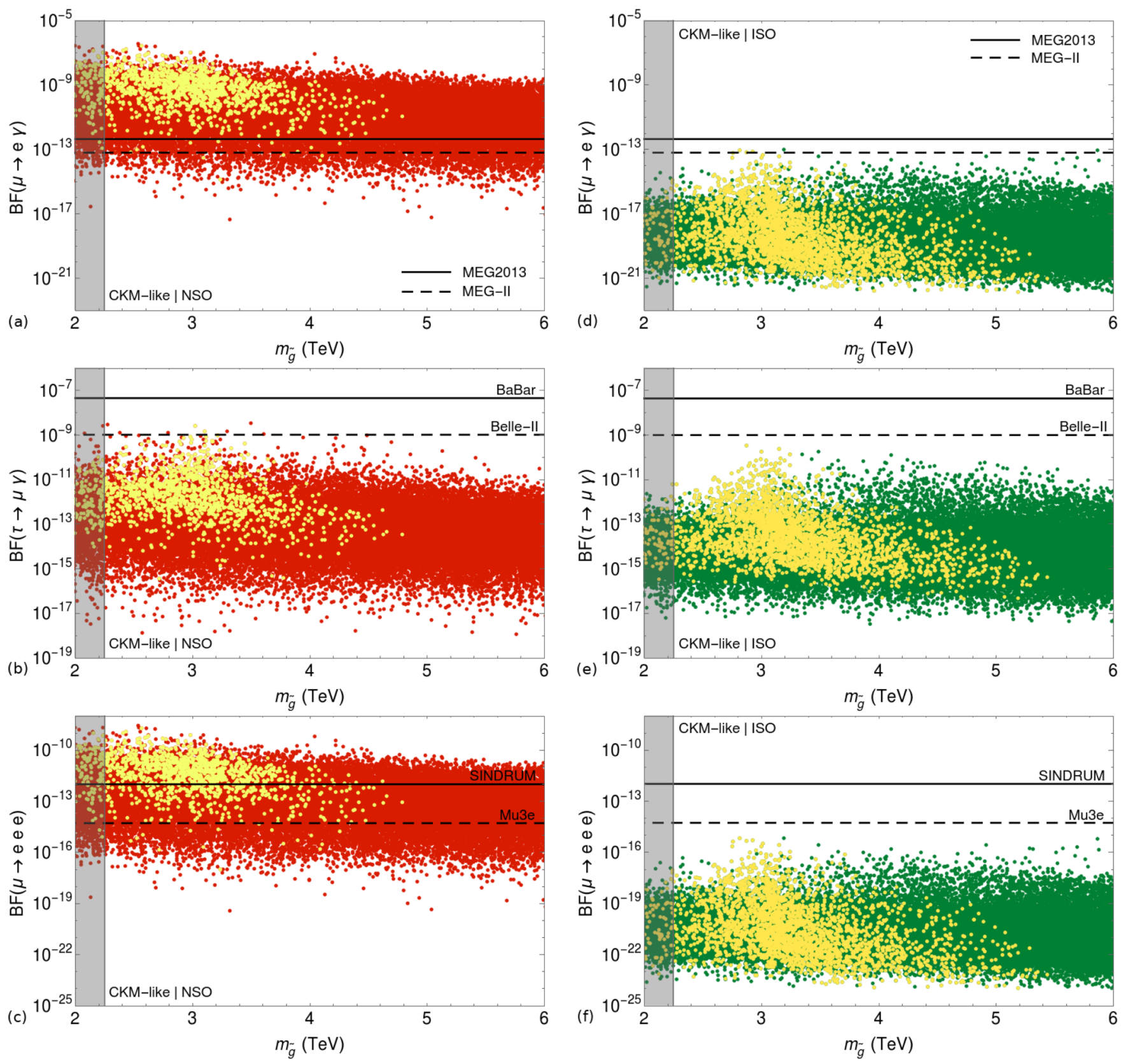

FIG. 6. LFV observables for NSO (left) and ISO (right) for the case No. 1 small (CKM-like) mixing scenario. Red points denote NSO while green points denote ISO. Yellow points are natural with $\Delta_{\mathrm{EW}}<30$. The gray shaded region is excluded by LHC run 2 gluino search constraints.

within the reach of future experiments compared to that of small CKM-like mixing. Similar to the CKM-mixing case, models with a NSO generate a few orders of magnitude larger rates compared to the ISO scenario.

For the large PMNS-like mixing scenario, the NSO is nearly completely ruled out by the MEG2013 results. The surviving points feature a heavy spectra for SUSY particles, $m_{\text {SUSY }} \gtrsim 10 \mathrm{TeV}$. For a heavier neutrino spectrum, LFV rates will be even more enhanced so our conclusions for the NSO case are valid for any set of neutrino masses with normal hierarchy $\left(m_{v_{3}} \gtrsim 0.05 \mathrm{eV}\right)$. Unlike the NUHM1 model results of Ref. [20], $\tan \beta$ values up to $\sim 50$ are still allowed for the NUHM3 model.
For the ISO case with large PMNS-like mixing, the MEG2013 limit rules out about half of the scanned parameter space points. The MEG-II experiment can probe more of, but not all of, the large mixing NUHM3 parameter space with an ISO.

Natural SUSY points show a similar behavior as in small CKM-like mixing case: smaller LFV rates for all decays in the ISO case as compared with the NSO. MEG-II and Mu3e searches can probe much of the NUHM3 large mixing ISO parameter space but are not sensitive enough to cover its entirety. Points over the entire range of gluino masses can escape from all search channels since in NUHM $3 \tan \beta$ values less than 20 are allowed and these predict small LFV rates. 

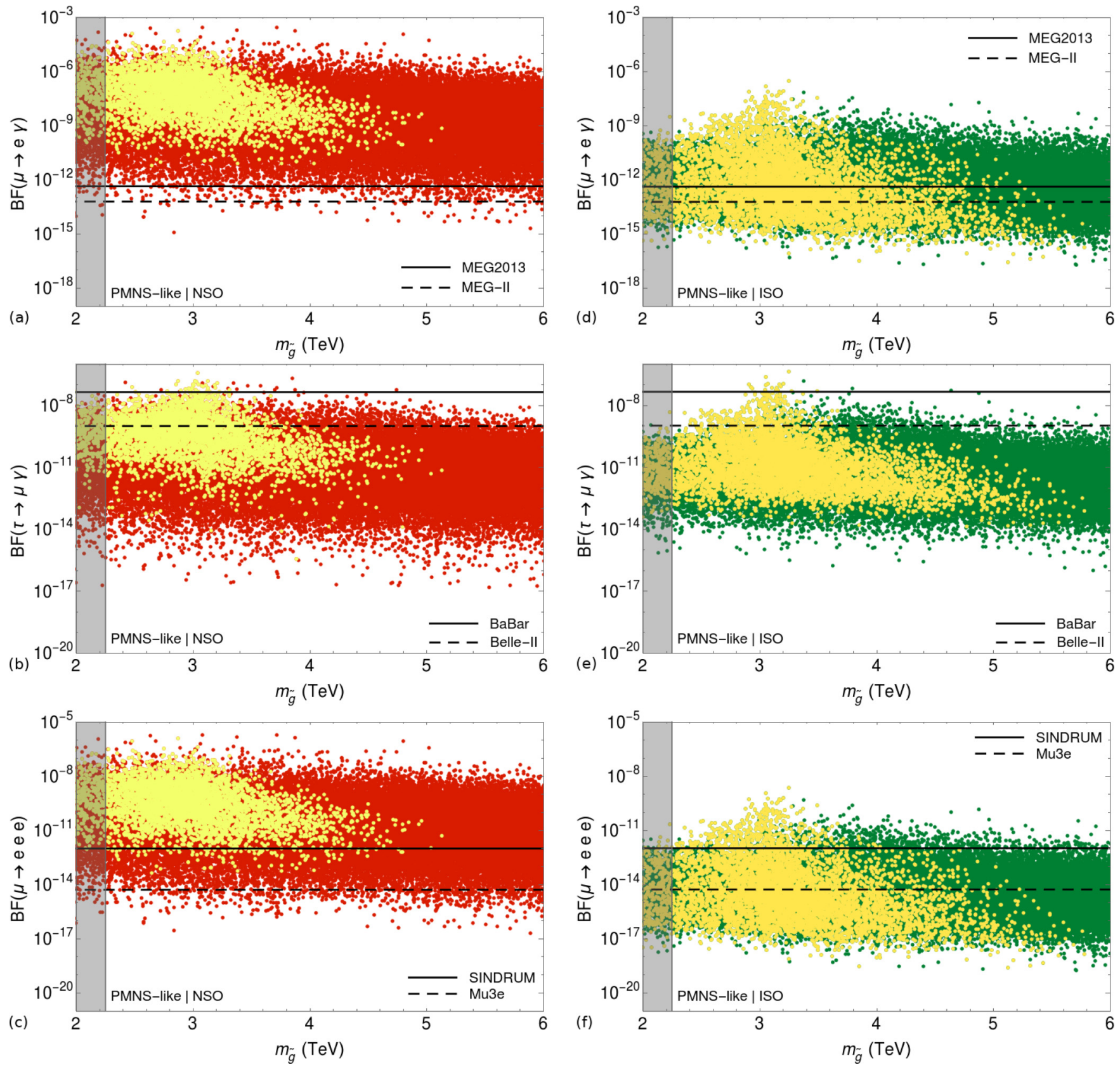

FIG. 7. LFV observables for NSO (left) and ISO (right) for the case No. 2 large (PMNS-like) mixing scenario. Red points denote NSO while green points denote ISO. Yellow points are natural with $\Delta_{\mathrm{EW}}<30$. The gray shaded region is excluded by LHC run 2 gluino search constraints.

\section{B. $\mu \rightarrow e$ conversion in nuclei}

In Fig. 8, we focus on $\mu N \rightarrow e N$ conversion in aluminum and titanium nuclei. The Mu2e experiment is planned to initially start with aluminum targets which can be replaced with titanium ones without any major changes in detectors [62]. Titanium targets are expected to increase the sensitivity by a factor of $\sim 1.6[53,63]$.

The projected initial sensitivity of $\mathrm{Mu} 2 \mathrm{e}, \mathrm{CR}(\mu+\mathrm{Al} \rightarrow$ $e+\mathrm{Al}) \sim 2.4 \times 10^{-17}$, is shown by the horizontal dashed line. The approximate sensitivity with the titanium target is shown by the vertical dashed line. We also mark the points that are within the reach of MEG-II searches (blue) and points that are already ruled out by MEG2013 (gray) on the same plane.
Only the points which are currently safe from LHC searches are shown in Fig. 8.

The $\mu N \rightarrow e N$ conversion rates for small CKM-like mixing are shown on the left side of Fig. 8. With the titanium target upgrade, the Mu2e experiment will probe almost the entire parameter space with NSO (red) but only a small portion of the parameter space predicted by models with the ISO (green). For the large PMNS-like mixing scenario (right side), even the initial run of Mu2e will cover all of the NSO parameter space and a large portion of the ISO parameter space. The Mu2e will reach sufficient sensitivity to cover the entirety of the large PMNS-like mixing parameter space for both the NSO and ISO cases with the titanium target 

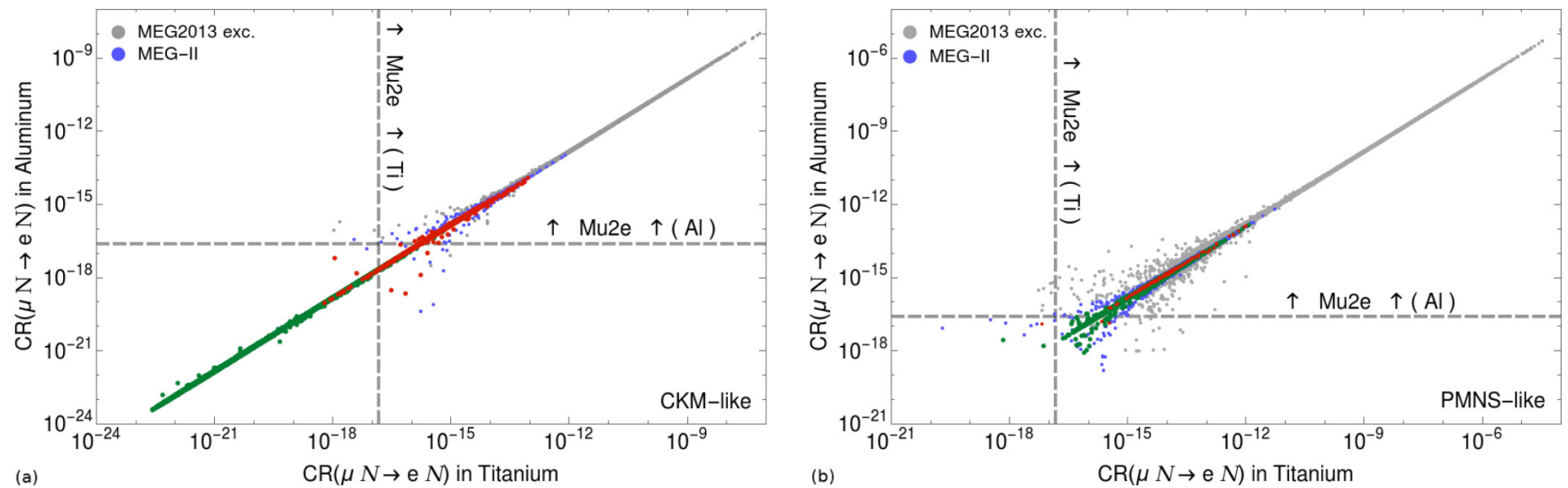

FIG. 8. Conversion rate (CR) for $\mu N \rightarrow e N$ in aluminum and in titanium nuclei with small CKM-like mixing (a) and large PMNS-like mixing (b) in SUSY SO(10) models with nonuniversal scalars. MEG2013 excluded points and points within MEG-II reach are colored in gray and blue, respectively. The dashed gray lines show the projected sensitivity of the Mu2e experiment. Red points denote the NSO while green points denote the ISO.

upgrade. The proposed Mu2e-II upgrade envisions to improve the sensitivity by an order of magnitude, $\mathrm{CR}(\mu+\mathrm{Al} \rightarrow e+$ $\mathrm{Al}) \sim 2.5 \times 10^{-18}$ [64], which is still not quite sufficient to cover the whole CKM-like mixing scenario with ISO.

\section{SUMMARY AND CONCLUSIONS}

The seesaw mechanism is highly motivated in that a large, intermediate mass scale for Majorana neutrinos explains the tiny active neutrino masses while also explaining the absence of right-handed neutrino effects in low-energy data. The seesaw mechanism is not a particularly well-motivated extension of the SM all by itself since the (apparently fundamental) newly discovered Higgs boson mass would likely blow up to the seesaw scale due to its quadratic divergences. Supersymmetry stabilizes the Higgs mass so the weak scale can coexist along with the Majorana mass scale (and the GUT and Planck scales). Within models containing a SUSY seesaw mechanism, then LFV processes should occur, possibly at an observable level. Our goal in this paper has been to present predictions for LFV processes within plausible SUSY models that are compatible with LHC run 2 results. These should include natural SUSY models with radiatively driven naturalness [24] which allow for a 125-GeV Higgs mass along with multi-TeV soft terms (as implied by LHC data) while at the same time avoiding the fine tunings associated with a little hierarchy: why is $m_{\text {weak }} \ll m_{\text {soft }}$ ?

We have investigated the current and projected reaches of various LFV search experiments within both the natural and unnatural portions of the NUHM3 SUSY model where neutrinos are generated with a type-I seesaw mechanism. We have limited the SUSY parameter space within the HE-LHC gluino reach. The NUHM3 model is well motivated in that it allows for weak scale naturalness along with a $125-\mathrm{GeV}$ Higgs mass and sparticles beyond LHC run 2 limits. The generational nondegeneracy is well motivated by both landscape [29] and minilandscape [55] string-motivated models which tend to give $m_{0}(3) \sim$ few $\mathrm{TeV}$ while $m_{0}(1,2) \sim$ tens of $\mathrm{TeV}$. We evaluated $t w o$ scenarios for the neutrino couplings: scenario
No. 1 small CKM-like mixing with $\mathbf{f}_{v}=\mathbf{f}_{u}$ and scenario No. 2 large PMNS-like mixing with $\mathbf{f}_{v}=\mathbf{f}_{u}^{\text {diag }} \mathrm{U}^{\mathrm{PMNS}}$. These scenarios are originally motivated by $4 d \mathrm{SO}(10)$ SUSY GUT models. We should remark here that the large mixing case arises from $16 \mathrm{~s}$ of $\mathrm{SO}(10)$ which couple to 120-dimensional Higgs representations. Such large Higgs representations do not appear in stringy constructions [42] and hence may well occupy the swampland [41]: theories inconsistent with string theory. Both the small and large mixing cases should be consistent with local GUT theories which can emerge from string theory [44]. Indeed, heterotic string models compactified on certain orbifolds can easily include the type-I SUSY seesaw while allowing for split multiplets which solve the doublettriplet splitting problem [43].

In our analysis, the third-generation neutrino mass is fixed to $0.05 \mathrm{eV}$ and then first- and second-generation neutrino masses are calculated using observed squared-mass differences. Along with large and small Yukawa mixing cases, we divided our results according to normal and inverted sneutrino mass orderings: NSO and ISO. Our calculations show that for natural SUSY with the NSO, rates for $\mathrm{BF}(\mu \rightarrow e \gamma)$ are already ruled out by the MEG2013 experiment for both small and large mixing scenarios. The $\mu \rightarrow e \gamma$ decay rate for the (landscape-favored) ISO scenario is lower than the NSO case due to the loop cancellations and decoupling effects. Even so, large chunks of parameter space for the ISO with large mixing are already ruled out, although substantial regions remain viable. In contrast, the LFV decay rates for the (string-favored) ISO with small CKM-like mixing have not yet been touched by LFV search experiments. Moreover, LFV predictions from these latter models lie below even the future attainable limits so it appears hard to be optimistic that meaningful constraints will be gained from LFV probes in the most plausible SUSY scenarios. In addition, the Mu2e conversion search experiment apparently can test (nearly) the entire parameter space of both NSO and ISO models with large mixing. The small mixing case for the ISO model remains with predicted detection rates well below the projected sensitivity of $\mu \rightarrow e$ conversion experiments. 

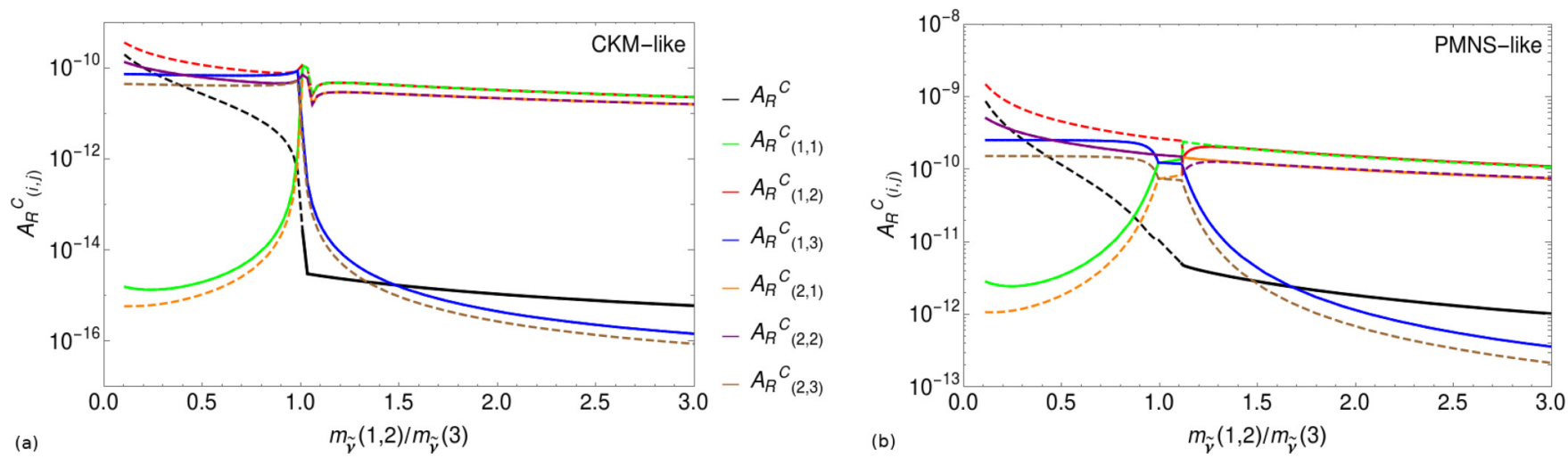

FIG. 9. Individual contributions to $A_{R}^{C}$ versus $m_{\tilde{v}}(1,2) / m_{\tilde{v}}$ (3). Dashed (solid) curves show negative (positive) amplitudes.

\section{ACKNOWLEDGMENTS}

This work was supported in part by the U.S. Department of Energy, Office of High Energy Physics under Contracts No. DE-SC0017647 and No. DE-SC0009956. The computing for this project was performed at the OU Supercomputing Center for Education \& Research (OSCER) at the University of Oklahoma (OU). The Feynman diagrams in Fig. 3 were drawn with JaxoDraw [65].

\section{APPENDIX A: NEUTRINO COUPLINGS AT $\boldsymbol{m}_{\mathrm{GUT}}$}

For neutrino couplings with CKM- and PMNS-like mixings, we consider the parametrizations

$$
\begin{aligned}
\left(\mathbf{f}_{v}\right)_{i j}^{\mathrm{CKM}} & =\left(\mathbf{f}_{u}\right)_{i j}=\left(\begin{array}{ccc}
Y_{u} & 0 & 0 \\
0 & Y_{c} & 0 \\
0 & 0 & Y_{t}
\end{array}\right)_{i k} \times V_{k j}^{\mathrm{CKM}} \\
& =\left(\begin{array}{ccc}
0.00000567 & 0.00000128 & 0.0000000128 \\
-0.000294 & 0.00130 & 0.0000536 \\
0.00279 & -0.0189 & 0.476
\end{array}\right)
\end{aligned}
$$

and

$$
\begin{aligned}
\left(\mathbf{f}_{v}\right)_{i j}^{\mathrm{PMNS}} & =\left(\mathbf{f}_{u}\right)_{i k}^{\mathrm{diag}} \times V_{k j}^{\mathrm{PMNS}} \\
& =\left(\begin{array}{ccc}
0.00000482 & 0.00000313 & 0.000000852 \\
-0.000659 & 0.000792 & 0.000861 \\
0.128 & -0.296 & 0.366
\end{array}\right)
\end{aligned}
$$

at GUT scale. $V^{\mathrm{CKM}}$ and $V^{\mathrm{PMNS}}$ are the Cabibbo-KobayashiMaskawa quark mixing and the Pontecorvo-Maki-NakagawaSakata neutrino mixing matrices, respectively. $Y_{u, c, t}$ denote Yukawa couplings of the up-type quarks. In the numerical expressions, we have set all complex phases to zero.

\section{APPENDIX B: LOOP CANCELLATIONS}

The decay width for the process $\mu \rightarrow e \gamma$ is given by

$$
\Gamma(\mu \rightarrow e \gamma)=\frac{e^{2}}{16 \pi} m_{\mu}^{5}\left(\left|A_{L}\right|^{2}+\left|A_{R}\right|^{2}\right),
$$

where $A_{L}=A_{L}^{N}+A_{L}^{C}$ and $A_{R}=A_{R}^{N}+A_{R}^{C}$ are the sum of the contributions from chargino $(C)$ and neutralino $(N)$ loop diagrams. The main contribution to $\mathrm{BF}(\mu \rightarrow e \gamma)$ comes from the chargino sector. Amplitudes of contributions to $A_{R}^{C}$ are shown in Fig. 9 with respect to the sneutrino mass ordering for both small CKM-like (a) and large PMNS-like (b) mixing cases. The individual contribution $A_{R(i, j)}^{C}$ where $i$ and $j$ are the indices for the chargino $\left(\chi_{1,2}^{ \pm}\right)$and sneutrino $\left(\tilde{v}_{1,2,3}\right)$ generations, respectively, are defined in Ref. [34]. Dashed lines represent negative amplitudes whereas solid lines are positive amplitudes. The black line shows the sum of all the individual contributions: $A_{R}^{C}$. For $m_{\tilde{v}_{3}}<m_{\tilde{v}_{1,2}}$, the amplitudes $A_{R(1,1)}^{C}$ and $A_{R(1,2)}^{C}, A_{R(2,1)}^{C}$ and $A_{R(2,2)}^{C}$ cancel out. As a result, $A_{R}^{C} \simeq A_{R(1,3)}^{C}+A_{R(2,3)}^{C}$ : one obtains a small amplitude contributing to the muon decay.
[1] A. Canepa, Rev. Phys. 4, 100033 (2019).

[2] E. Witten, Nucl. Phys. B 188, 513 (1981); R. K. Kaul, Phys. Lett. B 109, 19 (1982).

[3] H. Goldberg, Phys. Rev. Lett. 50, 1419 (1983); J. R. Ellis, J. S. Hagelin, D. V. Nanopoulos, K. A. Olive, and M. Srednicki, Nucl. Phys. B 238, 453 (1984).

[4] J. A. Casas and A. Ibarra, Nucl. Phys. B 618, 171 (2001); F. Deppisch, H. Pas, A. Redelbach, R. Ruckl, and Y. Shimizu, Nucl. Phys. Proc. Suppl. 116, 316 (2003).

[5] H. Baer and X. Tata, Weak Scale Supersymmetry: From Superfields to Scattering (Cambridge University Press, Cambridge, 2006); M. Drees, R. Godbole, and P. Roy, Theory and Phenomenology of Sparticles: An Account of Four-Dimensional
$N=1$ Supersymmetry in High Energy Physics (World Scientific, Hackensack, USA, 2004), p. 555; S. P. Martin, Adv. Ser. Direct. High Energy Phys. 21, 1 (2010); D. J. H. Chung, L. L. Everett, G. L. Kane, S. F. King, J. D. Lykken, and L. T. Wang, Phys. Rep. 407, 1 (2005).

[6] S. Dimopoulos, S. Raby, and F. Wilczek, Phys. Rev. D 24, 1681 (1981); U. Amaldi, W. de Boer, and H. Furstenau, Phys. Lett. B 260, 447 (1991); J. R. Ellis, S. Kelley, and D. V. Nanopoulos, ibid. 260, 131 (1991); P. Langacker and M. Luo, Phys. Rev. D 44, 817 (1991).

[7] L. E. Ibanez and G. G. Ross, Phys. Lett. B 110, 215 (1982); K. Inoue et al., Prog. Theor. Phys. 68, 927 (1982); 71, 413 (1984); L. Ibañez, Phys. Lett. B 118, 73 (1982); H. P. Nilles, 
M. Srednicki, and D. Wyler, ibid. 120, 346 (1983); J. Ellis, J. Hagelin, D. Nanopoulos, and M. Tamvakis, ibid. 125, 275 (1983); L. Alvarez-Gaumé, J. Polchinski, and M. Wise, Nucl. Phys. B 221, 495 (1983); B. A. Ovrut and S. Raby, Phys. Lett. B 130, 277 (1983); for a review, see L. E. Ibanez and G. G. Ross, C. R. Phys. 8, 1013 (2007).

[8] H. E. Haber and R. Hempfling, Phys. Rev. Lett. 66, 1815 (1991); J. R. Ellis, G. Ridolfi, and F. Zwirner, Phys. Lett. B 257, 83 (1991); Y. Okada, M. Yamaguchi, and T. Yanagida, Prog. Theor. Phys. 85, 1 (1991); M. Carena, M. Quiros, and C. E. M. Wagner, Nucl. Phys. B 461, 407 (1996); V. Barger, P. Huang, M. Ishida, and W. Y. Keung, Phys. Rev. D 87, 015003 (2013); for a review see, e.g., M. Carena and H. E. Haber, Prog. Part. Nucl. Phys. 50, 63 (2003).

[9] S. Heinemeyer, W. Hollik, D. Stockinger, A. M. Weber, and G. Weiglein, J. High Energy Phys. 08 (2006) 052.

[10] A. Baha Balantekin and B. Kayser, Ann. Rev. Nucl. Part. Sci. 68, 313 (2018).

[11] F. Capozzi, E. Di Valentino, E. Lisi, A. Marrone, A. Melchiorri, and A. Palazzo, Phys. Rev. D 95, 096014 (2017); I. Esteban, M. C. Gonzalez-Garcia, A. Hernandez-Cabezudo, M. Maltoni, and T. Schwetz, J. High Energy Phys. 01 (2019) 106.

[12] M. Tanabashi et al. (Particle Data Group), Phys. Rev. D 98, 030001 (2018).

[13] M. G. Aartsen et al., arXiv:1902.07771.

[14] M. A. Acero et al. (NOvA Collaboration), Phys. Rev. D 98, 032012 (2018).

[15] T. Deppisch, S. Schacht, and M. Spinrath, J. High Energy Phys. 01 (2019) 005.

[16] S. Vagnozzi, E. Giusarma, O. Mena, K. Freese, M. Gerbino, S. Ho, and M. Lattanzi, Phys. Rev. D 96, 123503 (2017).

[17] For a review, see, e.g., R. Arnowitt and P. Nath, in Perspectives on Supersymmetry II, edited by G. L. Kane (World Scientific, Singapore, 2010), pp. 222-243 and references therein; V. D. Barger, M. S. Berger, and P. Ohmann, Phys. Rev. D 47, 1093 (1993); 49, 4908 (1994); G. L. Kane, C. F. Kolda, L. Roszkowski, and J. D. Wells, ibid. 49, 6173 (1994).

[18] A. M. Baldini et al. (MEG Collaboration), Eur. Phys. J. C 76, 434 (2016).

[19] F. Renga (MEG Collaboration), Hyperfine Interact. 239, 58 (2018).

[20] L. Calibbi, D. Chowdhury, A. Masiero, K. M. Patel, and S. K. Vempati, J. High Energy Phys. 11 (2012) 040.

[21] K. Bora and G. Ghosh, Eur. Phys. J. C 75, 428 (2015).

[22] S. T. Petcov, W. Rodejohann, T. Shindou, and Y. Takanishi, Nucl. Phys. B 739208 (2006); D. N. Dinh, A. Ibarra, E. Molinaro, and S. T. Petcov, J. High Energy Phys. 08 (2012) 125; 09 (2013) 023(E); D. N. Dinh and S. T. Petcov, ibid. 09 (2013) 086.

[23] D. Matalliotakis and H. P. Nilles, Nucl. Phys. B 435, 115 (1995); M. Olechowski and S. Pokorski, Phys. Lett. B 344, 201 (1995); P. Nath and R. L. Arnowitt, Phys. Rev. D 56, 2820 (1997); J. Ellis, K. Olive, and Y. Santoso, Phys. Lett. B 539, 107 (2002); J. Ellis, T. Falk, K. Olive, and Y. Santoso, Nucl. Phys. B 652, 259 (2003); H. Baer, A. Mustafayev, S. Profumo, A. Belyaev, and X. Tata, J. High Energy Phys. 07 (2005) 065.

[24] H. Baer, V. Barger, P. Huang, A. Mustafayev, and X. Tata, Phys. Rev. Lett. 109, 161802 (2012).

[25] H. Baer, V. Barger, P. Huang, D. Mickelson, A. Mustafayev, and X. Tata, Phys. Rev. D 87, 115028 (2013).
[26] H. Baer, V. Barger, J. S. Gainer, D. Sengupta, H. Serce, and X. Tata, Phys. Rev. D 98, 075010 (2018)

[27] H. Baer, V. Barger, and D. Sengupta, Phys. Rev. D 98, 015039 (2018).

[28] X. Cid Vidal et al., arXiv:1812.07831.

[29] H. Baer, V. Barger, H. Serce, and K. Sinha, J. High Energy Phys. 03 (2018) 002.

[30] H. Baer, V. Barger, and H. Serce, Phys. Rev. D 94, 115019 (2016).

[31] K. J. Bae, H. Baer, A. Lessa, and H. Serce, J. Cosmol. Astropart. Phys. 10 (2014) 082; Front. Phys. 3, 49 (2015); H. Baer, A. Lessa, S. Rajagopalan, and W. Sreethawong, J. Cosmol. Astropart. Phys. 06 (2011) 031.

[32] H. Serce, AIP Conf. Proc. 1900, 040007 (2017); K. J. Bae, H. Baer, and H. Serce, J. Cosmol. Astropart. Phys. 06 (2017) 024.

[33] H. Fritzsch and P. Minkowski, Phys. Lett. B 62, 72 (1976); P. Minkowski, ibid. 67, 421 (1977); M. Gell-Mann, P. Ramond, and R. Slansky, Conf. Proc. C 790927, 315 (1979); T. Yanagida, ibid. 7902131, 95 (1979); R. N. Mohapatra and G. Senjanovic, Phys. Rev. Lett. 44, 912 (1980).

[34] J. Hisano, T. Moroi, K. Tobe, and M. Yamaguchi, Phys. Rev. D 53, 2442 (1996); J. Hisano and D. Nomura, ibid. 59, 116005 (1999).

[35] A. Masiero, S. K. Vempati, and O. Vives, Nucl. Phys. B 649, 189 (2003); L. Calibbi, A. Faccia, A. Masiero, and S. K. Vempati, Phys. Rev. D 74, 116002 (2006).

[36] T. Moroi, M. Nagai, and T. T. Yanagida, Phys. Lett. B 728, 342 (2014).

[37] M. Hirsch, F. R. Joaquim, and A. Vicente, J. High Energy Phys. 11 (2012) 105.

[38] V. Barger, D. Marfatia, A. Mustafayev, and A. Soleimani, Phys. Rev. D 80, 076004 (2009).

[39] A. Ilakovac, A. Pilaftsis, and L. Popov, Phys. Rev. D 87, 053014 (2013).

[40] F. Borzumati and A. Masiero, Phys. Rev. Lett. 57, 961 (1986).

[41] C. Vafa, arXiv:hep-th/0509212.

[42] J. Halverson and P. Langacker, PoS TASI 2017, 019 (2018)

[43] W. Buchmuller, K. Hamaguchi, O. Lebedev, S. RamosSanchez, and M. Ratz, Phys. Rev. Lett. 99, 021601 (2007).

[44] W. Buchmuller, K. Hamaguchi, O. Lebedev, and M. Ratz, arXiv:hep-ph/0512326.

[45] S. Antusch, J. Kersten, M. Lindner, M. Ratz, and M. A. Schmidt, J. High Energy Phys. 03 (2005) 024.

[46] S. T. Petcov, S. Profumo, Y. Takanishi, and C. E. Yaguna, Nucl. Phys. B 676, 453 (2004).

[47] A. M. Baldini et al. (MEG II Collaboration), Eur. Phys. J. C 78, 380 (2018).

[48] B. Aubert et al. (BaBar Collaboration), Phys. Rev. Lett. 104, 021802 (2010).

[49] K. Hayasaka (Belle Collaboration), Nucl. Phys. Proc. Suppl 225-227, 169 (2012); P. Branchini (Belle-II Collaboration), Universe 4, 101 (2018).

[50] E. Arganda and M. J. Herrero, Phys. Rev. D 73, 055003 (2006)

[51] U. Bellgardt et al. (SINDRUM Collaboration), Nucl. Phys. B 299, 1 (1988).

[52] A. K. Perrevoort (Mu3e Collaboration), SciPost Phys. Proc. 1, 052 (2019). 
[53] R. Kitano, M. Koike, and Y. Okada, Phys. Rev. D 66, 096002 (2002); 76, 059902(E) (2007).

[54] L. Bartoszek et al. (Mu2e Collaboration), arXiv:1501.05241.

[55] H. P. Nilles and P. K. S. Vaudrevange, Mod. Phys. Lett. A 30, 1530008 (2015).

[56] F. E. Paige, S. D. Protopopescu, H. Baer, and X. Tata, arXiv:hep-ph/0312045.

[57] D. Chowdhury, R. Garani, and S. K. Vempati, Comput. Phys. Commun. 184, 899 (2013).

[58] LEPSUSYWG, ALEPH, DELPHI, L3 and OPAL experiments, note LEPSUSYWG/yy-nn (http://lepsusy.web.cern.ch/lepsusy/ Welcome.html).
[59] M. Endo, T. Moroi, and M. M. Nojiri, J. High Energy Phys. 04 (2015) 176.

[60] E. Witten, Nucl. Phys. B 471, 135 (1996).

[61] M. Arana-Catania, E. Arganda, and M. J. Herrero, J. High Energy Phys. 09 (2013) 160; 10 (2015) 192(E).

[62] R. H. Bernstein (Mu2e Collaboration), Front. Phys. 7, 1 (2019).

[63] V. Cirigliano, R. Kitano, Y. Okada, and P. Tuzon, Phys. Rev. D 80, 013002 (2009).

[64] F. Abusalma et al. (Mu2e Collaboration), arXiv:1802.02599.

[65] D. Binosi, J. Collins, C. Kaufhold, and L. Theussl, Comput. Phys. Commun. 180, 1709 (2009). 